\title{
Polymorphisms of the vitamin D receptor gene and the risk of inflammatory bowel disease: a meta-analysis
}

\author{
L.Wang ${ }^{1 *}$, Z.T. Wang ${ }^{1 *}$, J.J. Hu ${ }^{2 *}$, R. Fan ${ }^{1}$, J. Zhou ${ }^{1}$ and J. Zhong ${ }^{1}$ \\ ${ }^{1}$ Department of Gastroenterology, Ruijin Hospital, \\ Shanghai Jiaotong University School of Medicine, Shanghai, China \\ ${ }^{2}$ Department of Nuclear Medicine, Ruijin Hospital, \\ Shanghai Jiaotong University School of Medicine, Shanghai, China \\ *These authors contributed equally to this study. \\ Corresponding author: J. Zhong \\ E-mail: Jimmyzj64@medmail.com.cn
}

Genet. Mol. Res. 13 (2): 2598-2610 (2014)

Received November 1, 2012

Accepted May 26, 2013

Published April 8, 2014

DOI http://dx.doi.org/10.4238/2014.April.8.2

\begin{abstract}
The gene encoding vitamin $\mathrm{D}$ receptor $(V D R)$ is recognized as a promising candidate for indicating the development of inflammatory bowel disease (IBD). Four genetic polymorphisms (ApaI, BsmI, FokI, TaqI) in $V D R$ have been widely evaluated to determine their association with IBD, and the results of these evaluations are often inconsistent. Therefore, we conducted a meta-analysis to shed some light on this issue and explored the sources of the heterogeneity between studies. We identified six articles for ApaI (cases/controls: 1902/1468), eight for TaqI (3053/2145), and five each for BsmI (1512/1616) and FokI (2315/1676). Data were analyzed under the random-effects model, and heterogeneity was explored by subgroup analyses. Overall, except for TaqI in allelic comparison [odds ratio $(\mathrm{OR})=0.90,95 \%$ confidence interval (CI): 0.83-0.98], ApaI, BsmI, and FokI polymorphisms showed no significant associations with IBD across different genetic models of inheritance. However, subgroup analyses indicated significance for the association of ApaI with Crohn's
\end{abstract}


disease (CD) risk (AA versus aa: $\mathrm{OR}=1.40 ; 95 \% \mathrm{CI}=1.05-1.88$ ), for $B s m \mathrm{I}$ in East Asians (BB plus $\mathrm{Bb}$ versus bb: $\mathrm{OR}=1.77,95 \% \mathrm{CI}=1.14-2.74$ ), for $\mathrm{Taq}$ I in Caucasians (TT plus Tt versus tt: $\mathrm{OR}=0.79,95 \% \mathrm{CI}=0.63$ 1.00 ), and with ulcerative colitis (UC) risk ( $\mathrm{T}$ versus t: $\mathrm{OR}=0.89,95 \% \mathrm{CI}$ $=0.80-0.99)$. There was a low probability of publication bias for all studied polymorphisms. Pooling previous individual studies on IBD, our findings demonstrated that the ApaI polymorphism may increase the risk of $\mathrm{CD}$, whereas the TaqI polymorphism may decrease the risk of UC, especially in Caucasians. Moreover, this study leaves open the question of divergent genetic profiles across different ethnic groups.

Key words: Vitamin D receptor; Polymorphism; Association study; Inflammatory bowel diseases; Meta-analysis

\section{INTRODUCTION}

Inflammatory bowel diseases (IBD) is a chronic relapsing inflammatory condition of the gastrointestinal tract and comprises two main subtypes, namely, Crohn's disease (CD) and ulcerative colitis (UC), that show overlapping but distinct clinical and pathological features. Although considerable efforts have been devoted to elucidating the etiology and pathogenesis of IBD, its underlying regulation and molecular mechanisms remain elusive. Epidemiological studies have documented that in comparison to the general population, the first-degree relatives of individuals with IBD carry about a 20 - to 50 -fold higher risk of developing CD and a 10 - to 20 -fold higher risk of developing UC (Zheng et al., 2003), indicating the genetic nature of IBD. Genomewide linkage analyses have identified multiple candidate regions on several chromosomes for IBD. Meanwhile, the loci of a number of immunity-related genes have been identified on several IBD-susceptibility regions (Hugot and Thomas, 1998; Hampe et al.,1998; Wild and Rioux, 2004). Considering the importance of immunity in IBD, investigations on the IBD-susceptibility genes involved in immunity have therefore attracted more attention. One candidate gene that is repeatedly studied in the pathogenesis of IBD is the vitamin D receptor (VDR) gene (12q12-14).

To date, four polymorphisms of the $V D R$ gene, which are recognized by the restriction enzymes ApaI (rs7975232), TaqI (rs731236), and BsmI (rs1544410) at the $3^{\prime}$ end and FokI (rs10735810) at the exon 2 of VDR gene (Morrison et al., 1994; Gross et al., 1996), have been exhaustively studied. However, the results of these studies are often irreproducible (Hughes et al., 2011). Individually underpowered association studies might have accounted for this disparity. In an effort to clarify the earlier inconclusive results, we decided to evaluate the association of VDR polymorphisms with the occurrence of IBD by a comprehensive meta-analysis of the current literature and to address the potential sources of heterogeneity and publication bias.

\section{METHODS}

\section{Search strategy for identification of studies}

We searched the PubMed, EMBASE, and ISI Web of Knowledge databases before August 12, 2012 by using the key subjects "inflammatory bowel disease", "IBD”, "Crohn's 
disease", "CD", "Ulcerative colitis", "UC", in combination with "vitamin D (1,25- dihydroxyvitamin D3) receptor" and "VDR". We read the full text of the retrieved articles to determine whether data on the topic of interest were included. Reference lists of the retrieved articles and systematic reviews were also screened for citations of articles that were not initially identified. Special meeting issues of journals (Abstract only) were removed from the search results. If more than one geographic or ethnic heterogeneous group was mentioned in one report, information on each was extracted separately. If two or more studies completely or partially shared study populations, the one with the larger sample size was extracted.

\section{Inclusion/Exclusion criteria}

Identified studies satisfied the following criteria: 1) evaluation of at least one studied polymorphism (ApaI, BsmI, FokI, or TaqI) in the VDR gene and the risk for IBD (CD and/or UC); 2) case-control or cross-sectional or nested case-control study design; 3) availability of genotype or allele counts of studied polymorphisms between patients and controls in order to estimate the odds ratio (OR) and its corresponding 95\% confidence interval (CI). Meanwhile, reported crude/adjusted ORs of the studied associations were also included.

Studies investigating the progression, severity, phenotype modification, and treatment response and survival- or family-based studies were excluded. Case reports, editorials, review articles, and non-English articles were also excluded.

\section{Extracted information}

The following data from each qualifying study were extracted independently and entered into separate databases by two authors (Z.T.W. and J.J.H): first author's last name, publication year, population ethnicity, study design, genotyping methods, VDR polymorphism evaluated, baseline characteristics of the study population, including age, gender, subtype of the disease, and the genotype or allele distributions in patients and controls. Any encountered discrepancies were adjudicated by a discussion and a $100 \%$ consensus was reached.

\section{Statistical analysis}

In this meta-analysis, we assessed the association of the ApaI, BsmI, TaqI, and FokI polymorphisms with IBD risk in allelic, homozygous, and dominant and recessive models, respectively. Crude OR and 95\% CI were calculated to compare differences of alleles or genotypes between patients and controls.

Deviation from the Hardy-Weinberg equilibrium was assessed with controls by using the Pearson $\chi^{2}$ test. The random-effects model using the DerSimonian \& Laird method was employed to bring the individual effect-size estimates together irrespective of between-study heterogeneity (Cohn and Becker, 2003). Heterogeneity was evaluated by the $I^{2}$ statistic, which represents the percentage of the observed between-study variability due to heterogeneity rather than chance, with values ranging $0-100 \%\left[I^{2}=0-25 \%\right.$, no heterogeneity; $I^{2}=25-50 \%$, moderate heterogeneity; $I^{2}=50-75 \%$, large heterogeneity; and $I^{2}=75-100 \%$, extreme heterogeneity] (Higgins et al., 2003). For between-study heterogeneity, we examined the study characteristics that could stratify the studies into subgroups with homogeneous effects. Subgroup analyses 
were conducted after stratifying studies performed on various ethnic/geographic populations or studies with different study designs or studies on different subtype diseases.

Finally, evidence for publication bias was assessed using Egger's test and visual funnel plot inspection. The Egger's test detects funnel plot asymmetry by determining whether the intercept deviates significantly from zero in a regression of the standardized effect estimates against their precision. We assessed publication bias on all the four considered genetic models.

Probability less than 0.05 was judged to be significant, with the exception of the $I^{2}$ statistic and publication tests, where a significance level of less than 0.1 was chosen. Statistical analyses were performed using STATA version 11.0 for Windows.

\section{RESULTS}

\section{Description of available studies}

The detailed selection process is presented in Figure 1. On the basis of the search rules, a total of 83 articles were initially identified. Further application of our inclusion/exclusion criteria yielded 9 articles (Simmons et al., 2000; Martin et al., 2002; Dresner-Pollak et al., 2004; Naderi et al., 2008; Noble et al., 2008; Pluskiewicz et al., 2009; Bentley et al., 2011; Hughes et al., 2011; Pei et al., 2011), which included 1666 cases of ulcerative colitis (UC) cases, 1796 cases of CD, and 2647 controls; these 9 articles were involved in this meta-analysis. There were 6 studies on $\mathrm{ApaI}$ (Simmons et al., 2000; Naderi et al., 2008; Noble et al., 2008; Pluskiewicz et al., 2009; Hughes et al., 2011; Pei et al., 2011), 5 on BsmI (Dresner-Pollak et al., 2004; Naderi et al., 2008; Pluskiewicz et al., 2009; Hughes et al., 2011; Pei et al., 2011) and FokI (Simmons et al., 2000; Naderi et al., 2008; Hughes et al., 2011; Pei et al., 2011; Bentley et al., 2011), and 8 on TaqI (Simmons et al., 2000; Martin et al., 2002; Naderi et al., 2008; Noble et al., 2008; Pluskiewicz et al., 2009 Hughes et al., 2011; Pei et al., 2011; Bentley et al., 2011), respectively. Altogether, there were 1902 (940 $\mathrm{CD} / 962 \mathrm{UC}$ ) cases and 1468 controls for polymorphism ApaI, 1512 (713 CD/799 UC) cases and 1616 controls for BsmI, 2315 (1098 CD/1217 UC) cases and 1676 controls for FokI, and 3053 $(1553 \mathrm{CD} / 1500 \mathrm{UC})$ cases, and 2145 controls for TaqI, respectively. The baseline characteristics of all qualifying studies are summarized in Table 1.

\section{ApaI and IBD}

Overall null significant association was found for ApaI polymorphism in allele comparison $(\mathrm{OR}=1.09,95 \% \mathrm{CI}=0.99-1.20)$, homozygote comparison $(\mathrm{OR}=1.16,95 \% \mathrm{CI}=$ $0.92-1.46)$, dominant model $(\mathrm{OR}=1.15,95 \% \mathrm{CI}=0.95-1.38)$, and recessive model $(\mathrm{OR}=$ $1.14 ; 95 \% \mathrm{CI}=0.93-1.41$ ) (Figure 2), even in subgroups by ethnicity and study design (Table 2). However, subgroup analyses by subtype disease suggested positivity for allele comparison $(\mathrm{OR}=1.17 ; 95 \% \mathrm{CI}=1.03-1.32)$ and homozygote model $(\mathrm{OR}=1.40 ; 95 \% \mathrm{CI}=1.05-1.88)$ with increased $\mathrm{CD}$ risk, even for under recessive models $(\mathrm{OR}=1.31 ; 95 \% \mathrm{CI}=1.06-1.63)$.

\section{BsmI and IBD}

We failed to find any significant association between $B s m$ I polymorphism and IBD risk in allele comparison $(\mathrm{OR}=1.11,95 \% \mathrm{CI}=0.96-1.29)$, homozygote comparison $(\mathrm{OR}=1.18,95 \% \mathrm{CI}$ 
$=0.95-1.47)$, dominant model $(\mathrm{OR}=1.12,95 \% \mathrm{CI}=0.92-1.38)$, and recessive model $(\mathrm{OR}=1.18$; $95 \% \mathrm{CI}=0.97-1.43)$, even in analyses stratified by study design or subtype disease. In ethnicitystratified analyses, a significant association was noted only among the population of East Asians in allele comparison $(\mathrm{OR}=1.67)$ and dominant model $(\mathrm{OR}=1.77,95 \% \mathrm{CI}=1.14-2.74)$.

\section{FokI and IBD}

No significance was noted in the association between FokI polymorphism and IBD risk in allele comparison $(\mathrm{OR}=0.87,95 \% \mathrm{CI}=0.71-1.07)$, homozygote comparison (OR $=0.65,95 \% \mathrm{CI}=0.40-1.07)$, dominant model $(\mathrm{OR}=0.69,95 \% \mathrm{CI}=0.46-1.04)$, and recessive model $(\mathrm{OR}=0.83 ; 95 \% \mathrm{CI}=0.58-1.19)$. Lack of association was identified in stratified analyses by study design or subtype disease. Meanwhile, we conducted an ethnicity-stratified analysis, and in general, there were obvious differences in risk estimates. For example, a decreased risk was observed across all genetic models in Middle Easterners; however, no material change in odds was observed in Caucasians or East Asians.

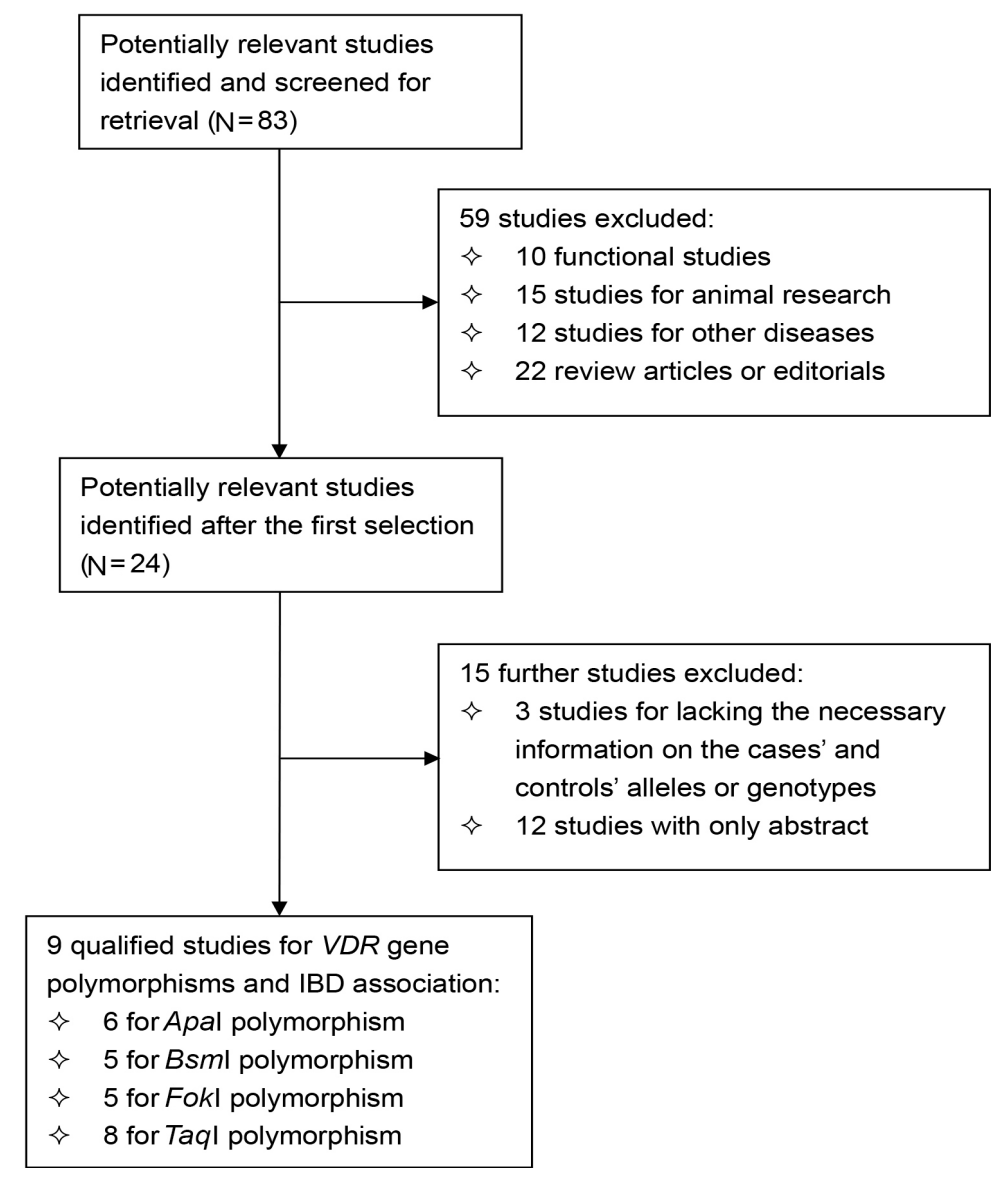

Figure 1. Flow diagram of search strategy and study selection. 


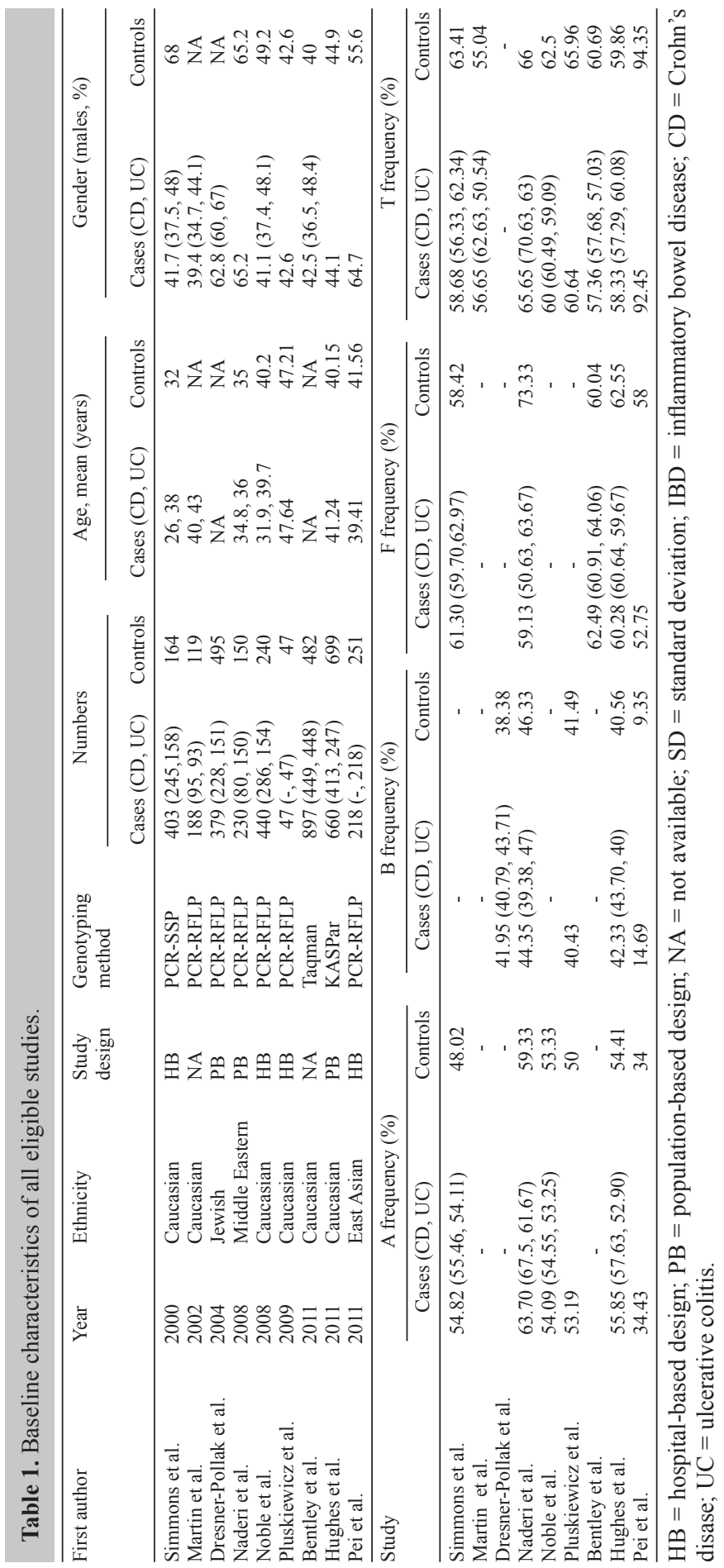



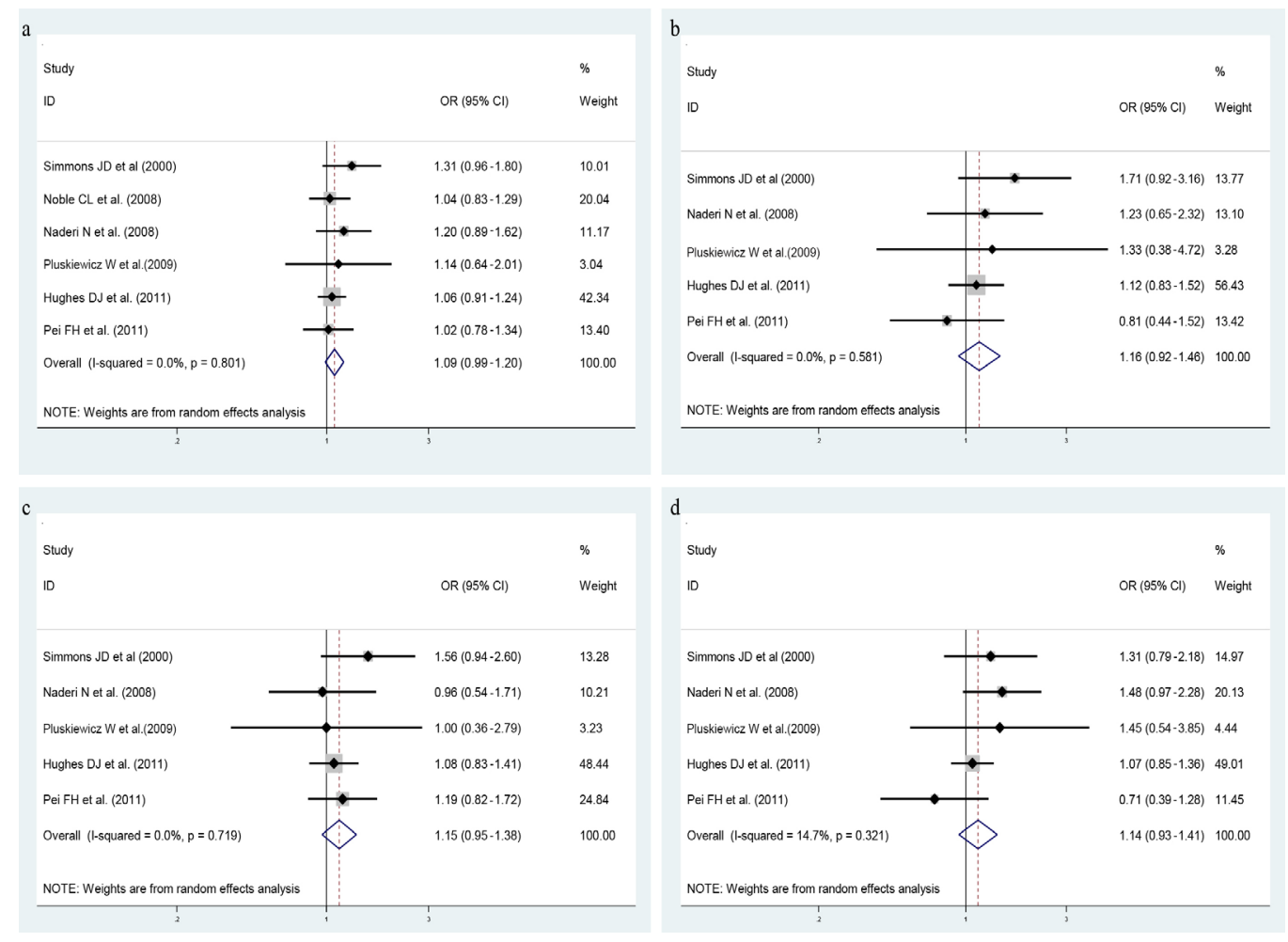

Figure 2. Association of ApaI polymorphism with IBD (a. allele comparison; b. homozygote model; c. dominant model; $\mathbf{d}$. recessive model).

\section{TaqI and IBD}

The pooled OR from all included studies indicated a significant association between $T a q \mathrm{I}$ polymorphism and IBD risk in allelic comparison $(\mathrm{OR}=0.90,95 \% \mathrm{CI}=0.83$ $0.98)$, without evidence of heterogeneity $\left(I^{2}=0.0 \% ; \mathrm{P}=0.859\right)$. While a marginally decreased risk was observed in the dominant model $(\mathrm{OR}=0.81,95 \% \mathrm{CI}=0.65-1.00)$, and no significant association was found in homozygote $(\mathrm{OR}=0.83,95 \% \mathrm{CI}=0.65-1.05)$ and recessive model $(\mathrm{OR}=0.95,95 \% \mathrm{CI}=0.82-1.11)$. In ethnicity-stratified analyses, there was a significant association among Caucasian populations in the allele model $(\mathrm{OR}=0.90)$, and a marginally decreased association was observed in the dominant model $(\mathrm{OR}=0.79$, $95 \% \mathrm{CI}=0.63-1.00)$, whereas no significance was observed in East Asians or Middle Eastern individuals in any kind of comparisons. Additional stratification by subtype disease showed an $11 \%$ decreased risk in UC cases $(95 \% \mathrm{CI}=0.80-0.99)$, which was negative in $\mathrm{CD}$ cases $(\mathrm{OR}=0.93,95 \% \mathrm{CI}=0.81-1.07)$ in allele comparison. Further stratification by study design showed a slightly decreased risk in hospital-based studies in comparison with population-based studies, with significance only identified in hospital-based studies under allele comparison and homozygote model. 


\section{Publication bias}

As reflected by the funnel plots (Figure 3) and the corresponding Egger test (Table 2), there was a low probability of publication bias for all polymorphisms examined.
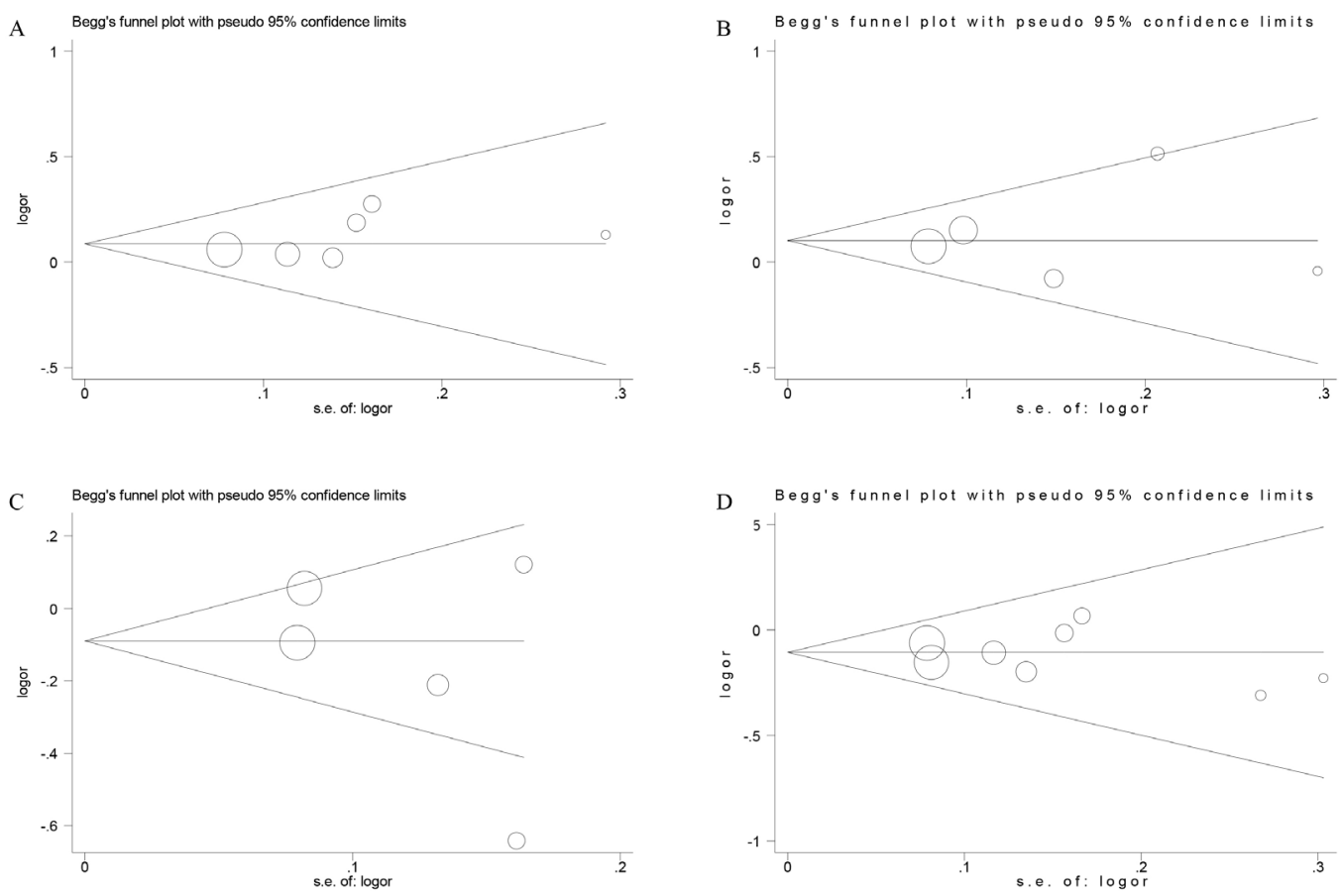

Figure 3. Funnel plots of allele comparisons for $V D R$ four polymorphisms. Vertical axis represents the log of OR; horizontal axis represents the SE of $\log (\mathrm{OR})$. Funnel plots are drawn with $95 \%$ confidence limits. OR $=$ odds ratio; $\mathrm{SE}=$ standard error. The graphic symbols represents the data in the plot be sized proportional to the inverse variance. A. VDR Apal allele comparison with IBD. B. VDR Bsml allele comparison with IBD. C. VDR Fokl allele comparison with IBD. D. VDR Taql allel comparison with IBD.

\section{DISCUSSION}

It has been over a decade since the first case-control study evaluating the VDR genetic variation and IBD was conducted (Simmons et al., 2000); however, the findings obtained subsequently remain conflicting. Herein, we quantitatively summarized all such studies to provide an evidence-based clue for the effectiveness of using the genetic markers located on the $V D R$ gene in determining susceptibility to IBD. Our results collectively suggested that the BsmI and FokI polymorphisms might not be genetic markers for IBD. However, subgroup analyses of the ApaI polymorphism revealed a significant association with CD subtype, while those of the TaqI polymorphism revealed a significant association with the UC subtype, especially in Caucasians. To the best of our knowledge, this meta-analysis was the first quantitative summarization of four well-characterized polymorphisms in the $V D R$ gene for their relevance to IBD. 


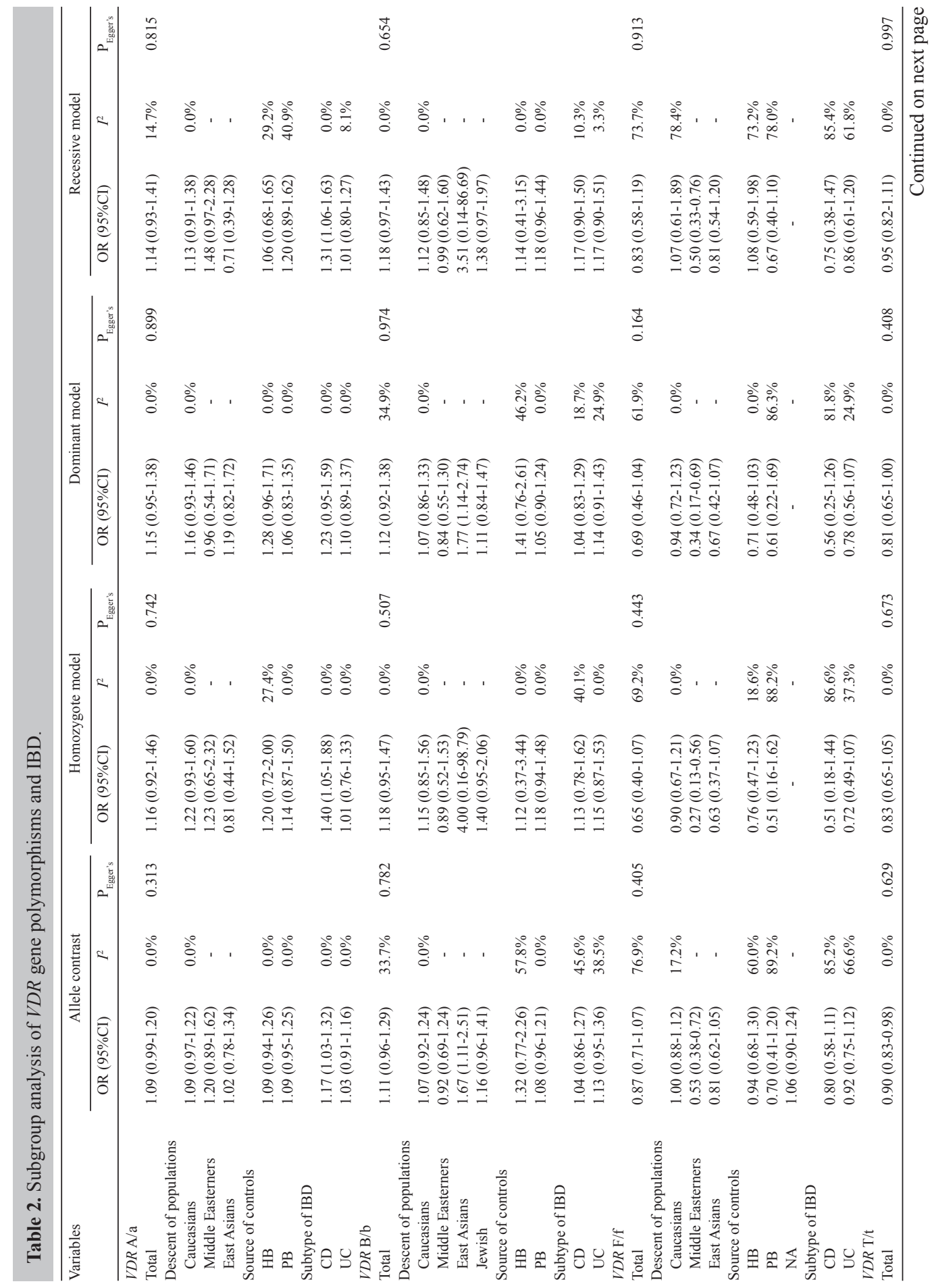


VDR Polymorphisms and IBD: A Meta-analysis

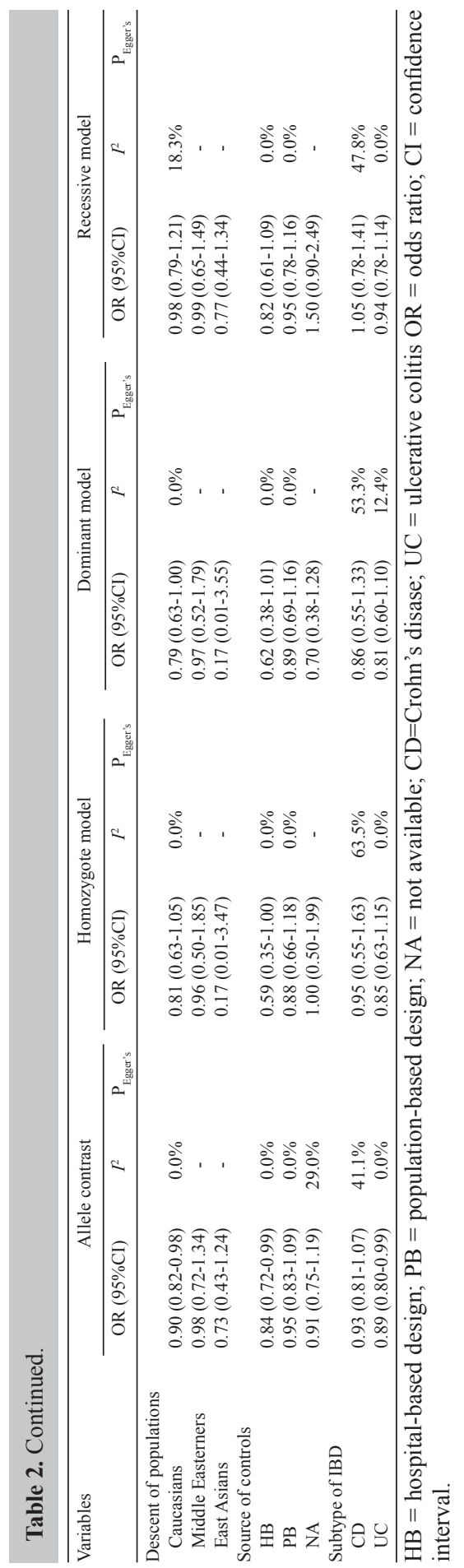


VDR is a member of the steroid receptor family that mediates the effects of vitamin D by regulating transcription of multiple cellular genes (Whitfield et al., 1995). The physiological actions of the VDR and its ligand are not only the well-known regulation of calcium and phosphorus uptake and transport, which are key processes controlling bone formation, but also their significant involvement in the control of immune functions (Hayes et al., 2003; Cantorna et al., 2004; Maruotti and Cantatore, 2010). VDR-knockout mouse studies showed that loss of VDR expression resulted in perturbed inflammation reactions in the gastrointestinal tract, leading to increased susceptibility to mucosal barrier damage and increased risk of IBD (Froicu et al., 2003; Froicu and Cantorna, 2007; Kong et al., 2008). Meanwhile, the gene encoding $V D R$ is located $3 \mathrm{cM}$ to the microsatellite marker D12S85 on chromosome 12, which has been reportedly linked to UC and CD (Satsangi et al., 1996; Curran et al., 1998; Duerr et al., 1998; Hampe et al., 1999). Therefore, the $V D R$ gene is regarded as a logical functional and positional candidate in susceptibility to IBD.

It is not uncommon to encounter genetic heterogeneity in any disease identification strategy (Hemminki et al., 2006). In this study, we speculate that the roles of VDR gene polymorphisms might vary across different ethnic populations. On one hand, there were striking differences in allele frequency between Caucasians and East Asians. For example, the frequency of the TaqI polymorphism $\mathrm{T}$ allele in Caucasians ranged from $55.04 \%$ to $65.96 \%$, which was remarkably lower than that in East Asians (94.35\%), indicating that different genetic backgrounds may cause this discrepancy or different populations may have different linkage disequilibrium patterns. Moreover, we cannot exclude the possibility that the VDR genetic markers may be in close linkage with different nearby causal polymorphisms in different populations. On the other hand, it is widely believed that complex diseases may have different genetic predispositions in people of different descents. For instance, genetic defects of CARD15/NOD2 have been strongly associated with CD in Caucasians (Nagy et al., 2005; Gazouli et al., 2005), while they were not associated with the disease in East Asian populations, including Japanese (Yamazaki et al., 2002) and Chinese (Leong et al., 2003) populations. Likewise, in our ethnicity-stratified analyses, IBD risk estimates associated with $V D R$ polymorphisms were remarkably different across ethnic groups, with Caucasians showing a positive association only for the TaqI polymorphism, but Middle Easterners showing a protective effect of FokI polymorphism. Similarly, for BsmI polymorphism, significance was merely found in East Asians, suggesting that the $V D R$ genetic defects might exert a pleiotropic role in the pathogenesis of IBD or interact with other genetic or environmental factors. However, considering the relatively small sample sizes in the East Asian and Middle Easterner populations, we agree that confirmation in large, well-designed studies is critical.

The two major subtypes of IBD, CD, and UC, are believed to have great differences in etiology and genetic backgrounds. For example, recent advances continue to highlight the abnormalities in innate immunity in CD patients, but not in UC (Marks, 2011). In subgroup analyses by IBD subtypes, we found that the ApaI polymorphism was associated with a significantly increased risk for $\mathrm{CD}$, but the TaqI polymorphism was associated with a protective effect against $\mathrm{UC}$, indicating the different roles of innate immunity and $V D R$ polymorphisms in the different IBD subgroups. Given the insufficient study power in each subgroup, much more research within the framework of genetics and biology is warranted.

This study has some limitations. First, all included studies were case-control in design, which precludes an inference on causality. Second, because only published studies were 
retrieved and articles in languages other than English were not included, publication bias might be possible, even though our funnel plots and statistical tests indicated no observable bias. Third, our results were based on unadjusted estimates. It seems likely that ApaI and TaqI can individually contribute to risk prediction in IBD patients, but whether these polymorphisms can be combined with other risk factors to enhance the prediction is a question that needs to be assessed in future studies. Thus, a more precise analysis should be conducted with individual data, which would allow for adjustment by other co-varieties such as age, gender, lifestyle, and other genetic factors.

In summary, we expand previous individual studies on IBD by indicating that the $V D R$ ApaI polymorphism may increase the risk of $\mathrm{CD}$, while TaqI polymorphism may decrease the risk of UC, especially in Caucasians. Moreover, this study leaves open the question of divergent genetic profiles across different ethnic groups. Nonetheless, for practical reasons, we hope that this study will not remain just another endpoint of research and instead serve as the first point to establishing background data for further investigation on the pathophysiological mechanisms of VDR genes on IBD.

\section{REFERENCES}

Bentley RW, Keown D, Merriman TR, Raj KM, et al. (2011). Vitamin D receptor gene polymorphism associated with inflammatory bowel disease in New Zealand males. Aliment. Pharmacol. Ther. 33: 855-856.

Cantorna MT, Zhu Y, Froicu M and Wittke A (2004). Vitamin D status, 1,25-dihydroxyvitamin D3, and the immune system. Am. J. Clin. Nutr. 80: 1717S-1720S.

Cohn LD and Becker BJ (2003). How meta-analysis increases statistical power. Psychol. Methods 8: 243-253.

Curran ME, Lau KF, Hampe J, Schreiber S, et al. (1998). Genetic analysis of inflammatory bowel disease in a large European cohort supports linkage to chromosomes 12 and 16. Gastroenterology 115: 1066-1071.

Dresner-Pollak R, Ackerman Z, Eliakim R, Karban A, et al. (2004). The BsmI vitamin D receptor gene polymorphism is associated with ulcerative colitis in Jewish Ashkenazi patients. Genet. Test. 8: 417-420.

Duerr RH, Barmada MM, Zhang L, Davis S, et al. (1998). Linkage and association between inflammatory bowel disease and a locus on chromosome 12. Am. J. Hum. Genet. 63: 95-100.

Froicu M and Cantorna MT (2007). Vitamin D and the vitamin D receptor are critical for control of the innate immune response to colonic injury. BMC Immunol. 8: 5.

Froicu M, Weaver V, Wynn TA, McDowell MA, et al. (2003). A crucial role for the vitamin D receptor in experimental inflammatory bowel diseases. Mol. Endocrinol. 17: 2386-2392.

Gazouli M, Mantzaris G, Kotsinas A, Zacharatos P, et al. (2005). Association between polymorphisms in the Tolllike receptor 4, CD14, and CARD15/NOD2 and inflammatory bowel disease in the Greek population. World J. Gastroenterol. 11: 681-685.

Gross C, Eccleshall TR, Malloy PJ, Villa ML, et al. (1996). The presence of a polymorphism at the translation initiation site of the vitamin D receptor gene is associated with low bone mineral density in postmenopausal Mexican-American women. J. Bone Miner. Res. 11: 1850-1855.

Hampe J, Hermann B, Bridger S, Macpherson AJ, et al. (1998). The interferon-gamma gene as a positional and functional candidate gene for inflammatory bowel disease. Int. J. Colorectal. Dis. 13: 260-263.

Hampe J, Schreiber S, Shaw SH, Lau KF, et al. (1999). A genomewide analysis provides evidence for novel linkages in inflammatory bowel disease in a large European cohort. Am. J. Hum. Genet. 64: 808-816.

Hayes CE, Nashold FE, Spach KM and Pedersen LB (2003). The immunological functions of the vitamin D endocrine system. Cell Mol. Biol. 49: 277-300.

Hemminki K, Lorenzo BJ and Forsti A (2006). The balance between heritable and environmental aetiology of human disease. Nat. Rev. Genet. 7: 958-965.

Higgins JP, Thompson SG, Deeks JJ and Altman DG (2003). Measuring inconsistency in meta-analyses. BMJ 327: $557-$ 560.

Hughes DJ, McManus R, Neary P, O’Morain C, et al. (2011). Common variation in the vitamin D receptor gene and risk of inflammatory bowel disease in an Irish case-control study. Eur. J. Gastroenterol. Hepatol. 23: 807-812.

Hugot JP and Thomas G (1998). Genome-wide scanning in inflammatory bowel diseases. Dig. Dis. 16: 364-369. 
Kong J, Zhang Z, Musch MW, Ning G, et al. (2008). Novel role of the vitamin D receptor in maintaining the integrity of the intestinal mucosal barrier. Am. J. Physiol. Gastrointest. Liver Physiol. 294: G208-G216.

Leong RW, Armuzzi A, Ahmad T, Wong ML, et al. (2003). NOD2/CARD15 gene polymorphisms and Crohn's disease in the Chinese population. Aliment. Pharmacol. Ther. 17: 1465-1470.

Marks DJ (2011). Defective innate immunity in inflammatory bowel disease: a Crohn's disease exclusivity? Curr. Opin. Gastroenterol. 27: 328-334.

Martin K, Radlmayr M, Borchers R, Heinzlmann M, et al. (2002). Candidate genes colocalized to linkage regions in inflammatory bowel disease. Digestion 66: 121-126.

Maruotti N and Cantatore FP (2010). Vitamin D and the immune system. J. Rheumatol. 37: 491-495.

Morrison NA, Qi JC, Tokita A, Kelly PJ, et al. (1994). Prediction of bone density from vitamin D receptor alleles. Nature 367: 284-287.

Naderi N, Farnood A, Habibi M, Derakhshan F, et al. (2008). Association of vitamin D receptor gene polymorphisms in Iranian patients with inflammatory bowel disease. J. Gastroenterol. Hepatol. 23: 1816-1822.

Nagy Z, Karádi O, Rumi G, Rumi G Jr., et al. (2005). Crohn's disease is associated with polymorphism of CARD15/ NOD2 gene in a Hungarian population. Ann. N. Y. Acad. Sci. 1051: 45-51.

Noble CL, McCullough J, Ho W, Lees CW, et al. (2008). Low body mass not vitamin D receptor polymorphisms predict osteoporosis in patients with inflammatory bowel disease. Aliment. Pharmacol. Ther. 27: 588-596.

Pei FH, Wang YJ, Gao SL, Liu BR, et al. (2011). Vitamin D receptor gene polymorphism and ulcerative colitis susceptibility in Han Chinese. J. Dig. Dis. 12: 90-98.

Pluskiewicz W, Zdrzalek J and Karasek D (2009). Spine bone mineral density and VDR polymorphism in subjects with ulcerative colitis. J. Bone Miner. Metab. 27: 567-573.

Satsangi J, Parkes M, Louis E, Hashimoto L, et al. (1996). Two stage genome-wide search in inflammatory bowel disease provides evidence for susceptibility loci on chromosomes 3, 7 and 12. Nat. Genet. 14: 199-202.

Simmons JD, Mullighan C, Welsh KI and Jewell DP (2000). Vitamin D receptor gene polymorphism: association with Crohn's disease susceptibility. Gut. 47: 211-214.

Whitfield GK, Hsieh JC, Jurutka PW, Selznick SH, et al. (1995). Genomic actions of 1,25-dihydroxyvitamin D3. J. Nutr. 125: 1690S-1694S.

Wild GE and Rioux JD (2004). Genome scan analyses and positional cloning strategy in IBD: successes and limitations. Best. Pract. Res. Clin. Gastroenterol. 18: 541-553.

Yamazaki K, Takazoe M, Tanaka T, Kazumori T, et al. (2002). Absence of mutation in the NOD2/CARD15 gene among 483 Japanese patients with Crohn's disease. J. Hum. Genet. 47: 469-472.

Zheng CQ, Hu GZ, Zeng ZS, Lin LJ, et al. (2003). Progress in searching for susceptibility gene for inflammatory bowel disease by positional cloning. World J. Gastroenterol. 9: 1646-1656. 